\title{
Bloqueos de nervio periférico. ¿Cuándo no?
}

\section{Peripheral nerve blocks. When do not?}

Dr. Carlos Ruiz Arévalo ${ }^{1}$.

1 Anestesiólogo. Servicio de Pabellones, Recuperación y CMA.

Hospital Clínico Metropolitano La Florida

Instructor Adjunto. División de Anestesiología. Escuela de Medicina

Pontificia Universidad Católica de Chile.

Autor Corresponsal:

Dr. Carlos Ruiz A.

Anestesiólogo. Servicio de Pabellones, Recuperación y CMA.

Hospital Clínico Metropolitano La Florida

Instructor Adjunto. División de Anestesiología. Escuela de Medicina

Pontificia Universidad Católica de Chile.

Dirección: Froilán Roa 6542. La Florida, 8240000, Chile.

Teléfono: 226121600

E-mail: carlosruizarevalo@gmail.com

ORCID ID: https://orcid.org/0000-0002-8318-6407

Palabras Clave: Bloqueo nervioso, Daño Neural, Anticoagulantes.

Key words: Nerve Block, Neural Injury, Anticoagulants.

\section{Perlas Clínicas}

- El tratamiento con antiagregantes plaquetarios o anticoagulantes es un riesgo para la aparición de hematoma en el sitio de punción de un bloqueo nervioso. Trae consigo un aumento de la morbimortalidad sobre todo en estructuras profundas.

- Los procedimientos pueden dividirse en tres categorías según el riesgo de sangrado o hematoma: alto, intermedio o bajo. En cada una de ellas, se puede recomendar un tiempo de ventana o suspensión para la terapia anticoagulante.

- Siempre debe ponderarse el riesgo versus el beneficio en pacientes sometidos a terapia anticoagulante y antiagregante, además de la experiencia del operador y medidas de seguridad al realizar un bloqueo nervioso.

- El trauma directo por la aguja, la toxicidad de los anestésicos locales, inyección a alta presión y daño isquémico por hematoma son, entre otros, mecanismos asociados al daño neural durante un bloqueo de nervio periférico

- La Diabetes Mellitus genera la polineuropatía periférica más común en la actualidad. Actualmente no se ha demostrado un aumento del daño neural tras un bloqueo de nervio periférico en pacientes diabéticos. Sin embargo, la duración del bloqueo y eventualmente la toxicidad del anestésico local, podrían verse aumentadas.

- Otras neuropatías (EM, CMT, NF, MG) no han demostrado empeorar sus síntomas al realizar bloqueos 
de nervio periféricos. Sin embargo, los reportes de casos son escasos y falta evidencia para realizar recomendaciones definitivas.

- Actualmente las guías internacionales de la ASRA y ESRA, recomiendan que los bloqueos de nervio periférico no se realicen de rutina bajo sedación profunda o anestesia regional. Sin embargo, puede ser razonable plantear en casos seleccionados realizarlos bajo sedación profunda o anestesia general en operadores entrenados, con altas medidas de seguridad, neurolocalización con ultrasonido y ponderando riesgo versus beneficios.

- El consentimiento informado y la entrega de información precisa de los beneficios y potenciales riesgos es fundamental al momento de aplicar una técnica de anestesia regional periférica. El anestesiólogo y las instituciones en donde se apliquen estas técnicas, deberán buscar las condiciones de seguridad mas óptimas para el paciente.

\title{
Resumen
}

Los bloqueos de nervio periférico se han convertido en una técnica anestésica única o complementaria de gran utilidad para el manejo del perioperatorio. Su aplicación no está exenta de riesgos y complicaciones que deben evaluarse caso a caso. Tradicionalmente el uso concomitante de anticoagulantes o el daño neural se consideran una contraindicación para su aplicación rutinaria. Gran parte de las revisiones actuales en la literatura, entregan guías para evaluación del riesgo para la anestesia neuroaxial con poco énfasis en la anestesia regional periférica. La presente revisión pretende recopilar las recomendaciones actuales donde podría ser razonable evitar o diferir un bloqueo de nervio periférico.

\begin{abstract}
Peripheral nerve blocks have become a unique or complementary anesthetic technique that is very useful for perioperative management. Its application is not exempt from risks and complications that must be evaluated case by case. Traditionally, the concomitant use of anticoagulants or previous neural damage are considered contraindication for their routine use. Most of the current reviews in the literature provide guidelines for risk assessment for neuraxial anesthesia with little emphasis on peripheral regional anesthesia. This review aims to compile the current recommendations where it might be reasonable to avoid or defer a peripheral nerve block.
\end{abstract}

\section{Introducción}

El uso de la anestesia regional como técnica anestésica ha aumentado considerablemente en los últimos años, conforme se ha objetivado sus efectos secundarios mínimos, una rápida recuperación, un control adecuado del dolor postoperatorio y una menor estancia hospitalaria en el caso de pacientes ambulatorios ${ }^{(1)}$.

No obstante, esta técnica no está exenta de contraindicaciones y riesgos que tanto los servicios clínicos, como los propios operadores e incluso los pacientes deben considerar para lograr un procedimiento lo más seguro posible y con la mayor tasa de éxito ${ }^{(2)}$. Los esfuerzos de las distintas sociedades científicas se han centrado en generar las recomendaciones que permitan guiar a los anestesiólogos a realizar procedimientos de anestesia regional periférica en las condiciones más seguras para los pacientes y así mininmizar el riesgo de daño tras aplicar un bloqueo nervioso. Debido al importante impacto en la morbimortalidad, en las implicancias funcionales y medico-legales que puede tener un daño neurológico transitorio o permanente, es que persiste imperativo conocer por quienes practican estas técnicas, las potenciales contraindicaciones y situaciones clínicas donde una técnica regional puede ser desaconsejada. En la literatura actual, existe escasez de publicaciones que revisen en detalle las contraindicaciones a la anestesia regional periférica, no así las potenciales complicaciones que derivan de su práctica rutinaria.

La presente revisión tiene por intención reunir las actuales recomendaciones que permitan orientar al anestesiólogo general y especialista, en aquellas situaciones que tradicionalemente pueden contraindicar un bloqueo de nervio periférico.

\section{Antiagregación plaquetaria y Anticoagulantes:}

El uso de antiagregantes plaquetarios y anticoagulantes en pacientes ambulatorios y hospitalizados está cada vez mas extendido en la práctica cotidiana. El creciente aumento de las comorbilidades, la edad avanzada y el riesgo de trom- 
bosis venosa, hace que sea necesaria la profilaxis de un evento tromboembólico durante el periodo perioperatorio (3). Numerosas sociedades científicas a lo largo del mundo han realizado esfuerzos por reunir la evidencia existente para orientar a los anestesiólogos en las precauciones y restricciones al uso de técnicas regionales en pacientes sometidos a antiagregación plaquetaria y/o anticoagulación ya sea profiláctica o terapéutica. Sin embargo, debido a la importante morbimortalidad que genera una complicación neuroaxial $(3,4)$, las guías clínicas existentes han centrado sus esfuerzos en reunir la mejor evidencia posible y sus recomendaciones a la anestesia neuroaxial, dejando de lado en gran parte los procedimientos regionales periféricos; esto ha llevado a que muchas de las directrices clínicas que existen en la actualidad sean sólo una extrapolación de la anestesia neuroaxial a la anestesia regional periférica. Diversas sociedades científicas han planteado la necesidad de contar con guías clínicas que diferencien las recomendaciones entre los procedimientos neuroaxiales y periféricos $(5,6,7)$

El hematoma en el sitio de punción o en las estructuras adyacentes a un catéter de nervio periférico, es una complicación que puede acarrear consigo 3 principales riesgos: necesidad de exploración quirúrgica para drenaje, transfusión y potencial daño neurológico por compresión ${ }^{(8)}$. El tratamiento con antiagregantes plaquetarios o anticoagulantes es un riesgo para la aparición de hematoma $(10,11)$. Si bien, el ultrasonido ha demostrado una disminución en el riesgo de punción de estructuras vasculares, en la gran mayoría de los casos reportados en la literatura con complicaciones hemorrágicas de la anestesia regional, no está informada la punción vascular accidental ${ }^{(7)}$. Actualmente, existen grupos de trabajo en anestesia regional que han planteado dividir los bloqueos de nervio periférico en tres grupos según el potencial de riesgo $(7,8,9)$ :

1 Bajo riesgo de sangrado: Son aquellos en los cuales, de ocurrir un evento hemorrágico, pueden ser sitios compresibles de fácil manejo. Incluye principalmente a bloqueos y estructuras superficiales. Estos procedimientos pueden realizarse concomitantemente con anticoagulación y antiagregación plaquetaria siempre que el beneficio supere al potencial riesgo del procedimiento y considere un operador experto ayudado de ultrasonido.

2 Riesgo Intermedio de sangrado: Son aquellos en que la posibilidad de sangrado y por ende complicaciones, es una posibilidad real y debe analizarse caso a caso anteponiendo riesgos versus beneficios. Puede incluir a estructuras superficiales o no y la realización de estos bloqueos deben ser manejadas por especialistas entrenados con todos los elementos de monitorización disponibles, incluida la neurolocalización bajo ultrasonido.

3 Alto riesgo de sangrado: Son aquellos ubicados en estructuras profundas no compresibles o bien en aquellas cuyo acceso es difícil y una complicación hemorrágica es potencialmente peligrosa. En estos bloqueos, deberá considerarse la farmacología de cada agente anticoagulante o antiagregantes para determinar ventana más óptima sin anticoagulación para su ejecución. Siempre se debe evaluar la relación riesgo versus beneficio y deben ser realizados por especialistas con entrenamiento y experiencia suficientes.

4 Vale la pena señalar que esta división es mas bien arbitraria y se basa en casos anecdóticos de complicaciones hemorrágicas en bloqueos profundos en pacientes sin anticoagulación y en aquellos que la recibieron; la reciente guía de la Sociedad Americana de Anestesia Regional (ASRA) considera los pocos casos documentados desde 1984 pero que son insuficientes para realizar recomendaciones definitivas ${ }^{(7)}$. Además, existen guías clínicas que incluyen al bloqueo supraclavicular e interescalénico como estructuras de mayor riesgo por el acceso difícil y el potencial riesgo de trauma vascular, aunque anatómicamente sean estructuras mas bien superficiales $(6,8)$. B. Tsui en conjunto con la Sociedad Canadiense de Anestesia (CAS) ${ }^{(9)}$, realizaron una revisión sistemática y consenso de expertos del riesgo de sangrado de los bloqueos de nervio periférico y bloqueos de planos interfasciales. En ella, proponen una clasificación más extensiva de los procedimientos regionales en las tres categorías antes mencionadas, aunque los riesgos no estén medidos necesariamente en pacientes que utilizan terapia anticoagulante. Ante la constante renovación y adición de nuevas técnicas de anestesia regional, resulta difícil clasificarlas todas según el riesgo potencial de sangrado; es necesaria mayor evidencia y de mejor calidad que permita diferenciar el riesgo real de complicaciones hemorrágicas en las nuevas técnicas de anestesia 
regional periférica y quedará mas bien a criterio del operador basado en su experiencia y entrenamiento determinar cuales de las noveles técnicas reviste mayor o menor riesgo de hematoma.

La Sociedad Europea de anestesia en su guía de práctica clínica del 2010 y la última actualización de la guía de la ASRA del $2018^{(6,7)}$, refuerzan el concepto del aumento de la morbimortalidad tras la aparición de un hematoma, sobre todo en estructuras profundas. Es por esto que también recomiendan diferenciar entre procedimientos de alto, intermedio y bajo riesgo de hematoma al momento de aplicar una técnica regional periférica. Por otro lado, estas guías clínicas son más específicas en señalar que las recomendaciones para la inserción y retiro de catéteres de nervio periférico deben ceñirse a aquellas vigentes para los catéteres neuroaxiales, siendo especialmente importante durante el retiro, toda vez que no existe justificación para remover un catéter durante el máximo efecto de un anticoagulante $(10,12)$. Las tablas 1 y 2 resumen la categorización de riesgo de cada procedimiento y las recomendaciones de tiempo de suspensión para anestesia regional periférica según estratificación de riesgo.

\section{Anestesia regional en daño neurológico previo:}

La neuropatía periférica es una condición clínica y subclínica, con una alta prevalencia en la población general. Se estima que está presente entre un $2 \%-8 \%$ de la población general, incrementándose en un $26 \%$ en pacientes con diabetes tipo 2 y en un $58 \%$ en pacientes con diabetes tipo 1 establecida ${ }^{(13)}$. A pesar de las ventajas que puede proveer una técnica regional periférica para el manejo del post operatorio, desde hace décadas existe una legítima aprehensión por parte de los anestesiólogos a utilizar anestesia regional en pacientes con daño neurológico previo; considerándola muchas veces una contraindicación a cualquier técnica regional con el fin de no empeorar el déficit previo al procedimiento quirúrgico.

El nervio periférico es una estructura frágil y expuesta al riesgo de lesiones. Es una proyección del sistema nervioso central que lleva información desde y hacia todo el organismo, formado por una compleja estructura de tejido neural, conectivo y delgados vasos sanguíneos que mantienen el funcionamiento metabólico del nervio (14). El mecanismo del potencial daño neurológico asociado a la anestesia regional ha sido estudiado en experiencias in vitro e in vivo, identificándose múltiples factores que lo influencian ${ }^{(15)}$ : Trauma directo causado por la aguja, toxicidad directa por anestésico local, daño isquémico por alta presión y volumen del anestésico local o adición de vasoconstrictores, hematoma o injuria vascular, factores intraoperatorios, injuria por torniquete y factores post operatorios incluyendo posicionamiento y edema. Todos estos factores están involucrados de una u otra manera al momento de realizar un bloqueo de nervio periférico.

Estudios prospectivos han estimado que la incidencia de injuria neurológica a largo plazo después de un bloqueo de nervio periférico se sitúa en un rango entre 2,4 a 4 por cada 10.000 bloqueos, mientras que déficits neurológicos transitorios hasta por 15 días pueden elevar su frecuencia hasta un $15 \%$ dependiendo de la serie estudiada ${ }^{(16)}$. Recientemente, Lam y colaboradores $(17)$ tras analizar una cohorte retrospectiva de 19.219 pacientes sometidos a anestesia regional periférica, identificaron una incidencia de síntomas neurológicos transitorios en un $14,4 \%$ y de síntomas prolongados en 1 en 1000 casos.

Upton y McComas en 1973 describieron el fenómeno de la doble injuria o "double-crush", el cual sugiere que los pacientes con compromiso neural preexistente pueden ser más susceptibles a una injuria cuando son expuestos a un segundo daño, aún si es otro lugar anatómico distinto a lo largo de la vía de transmisión neural. Este segundo daño puede incluir, entre otros, un bloqueo de nervio periférico. Así, la aplicación de una técnica regional en pacientes con daño neurológico preexistente podría teóricamente, posicionarlos en un elevado riesgo de sufrir una doble injuria y mayor déficit. Es importante señalar que, si bien la mayoría de los análisis retrospectivos o prospectivos de casos de daño neurológico asociado a anestesia regional periférica han asociado causas multifactoriales y sugieren que un daño neurológico previo puede acentuarse tras una nueva injuria neurológica, no existe la evidencia suficiente que permita asociar directamente el daño neural previo como un factor de riesgo para realizar déficit neurológico posterior a una técnica regional $(18,19)$. El examen clínico y la caracterización del déficit previo neurológico previo a realizar un bloqueo de nervio periférico en un paciente de riesgo junto a una detallada explicación de los potenciales efectos secundarios que pueden estar asociados, ponderando riesgo versus beneficios, es clave al momento de decidir por parte del anestesiólogo y del paciente si someterse a una técnica regional periférica o no ${ }^{(20)}$. Basadas en la 
evidencia disponible, se describirán algunas recomendaciones para las patologías neurológicas más habituales y su relación anestesia regional periférica.

\section{Diabetes Mellitus}

Actualmente es la causa más común de polineuropatía sistémica, con múltiples formas de presentación con la polineuropatía distal simétrica sensoriomotora como la más frecuente. A pesar del hecho de que muchos de quienes la presenten se mantengan asintomáticos, prácticamente todos tendrán evidencia de anormalidades en la conducción nerviosa y casi un 50\% de los pacientes con diabetes de larga data, tendrán síntomas de daño neurológico (21). Fisiopatológicamente la diabetes mellitus (DM) produce alteraciones en el metabolismo celular del nervio periférico asociados al estrés oxidativo, inflamación y disfunción mitocondrial por la prolongada exposición a la hiperglicemia. Este fenómeno lleva al aumento de la producción de radicales libres que inducen daño neuronal. El daño celular no es el único mecanismo patogénico asociado a la DM, recientes investigaciones experimentales han demostrado un daño precoz en la microvasculatura de los nervios sometidos a hiperglicemia, generando áreas de isquemia local. La suma estos mecanismos explicarían una disminución de la velocidad de conducción neuronal y teóricamente un aumento del riesgo de daño ${ }^{(22)}$.

Desde el punto de vista clínico, se han estudiado las principales consecuencias del daño generado por la DM en los nervios periféricos. En estudios experimentales y descriptivos $(23,24,25)$ se ha logrado establecer que el umbral de estimulación eléctrica en nervios periféricos con DM puede ser más alto del habitual con una variabilidad interindividual muy difícil de predecir, por lo que la neurolocalización como método de localización puede ser imprecisa e inducir a inyecciones intraneurales inadvertidas. Por otro lado, la sensibilidad de los nervios periféricos a los anestésicos locales está aumentada y la duración de los bloqueos también, presumiblemente por una disminución de flujo sanguíneo de la red de vasa nervorum y del metabolismo del nervio periférico. Esto puede llevar a un aumento de la toxicidad local del nervio periférico por lo que se recomienda disminuir las dosis de anestésico local al mínimo posible, tanto en concentración como en masa de droga. A pesar de estas asociaciones fisiopatológicas válidas, no existe evidencia suficiente que demuestre un aumento per se o aparición de daño neurológico sólo por el simple de hecho de tener DM y recibir una técnica de anestesia regional periférica, por lo que no lo contraindica de manera absoluta (26). Es recomendable así, disminuir las dosis de anestésico local al mínimo posible, evitar la adición de vasoconstrictores, preferir una técnica de neurolocalización guiada por ultrasonido y ponderar con el paciente los riesgos versus beneficios ante el potencial aumento transitorio de los síntomas neurológicos preoperatorios.

\section{Esclerosis múltiple}

La esclerosis múltiple (EM) es una enfermedad inflamatoria crónica desmielinizante del sistema nervioso central. Los síntomas principales cuenta, entre otros, alteraciones de la visión, déficit sensitivo, debilidad de extremidades, ataxia y disfunción del sistema digestivo-urinario. Afecta predominantemente a mujeres y hasta un $80 \%$ de los pacientes pueden tener un curso de la enfermedad con períodos de remisión. Sus causas incluyen una compleja interacción entre factores ambientales, inmunológicos y genéticos.

Existen reportes anecdóticos de anestesia regional periférica en pacientes con EM, donde se han descrito daño de plexo braquial asociado a bloqueo interescalénico y pérdida de la sensibilidad de extremidades después de bloqueos paravertebrales $(27,28)$. Actualmente no existen guías formales que contraindiquen un bloqueo de nervio periférico en pacientes con EM y sólo se hace énfasis en disminuir las dosis de anestésico local, evitar el uso de vasoconstrictores y utilizar una técnica basada en ultrasonido ponderando riesgos versus beneficios con el paciente ${ }^{(19,22)}$.

\section{Enfermedad de Charcot-Marie-Tooth}

La enfermedad de Charchot-Marie-Tooth (CMT) es la neuropatía periférica hereditaria más frecuente, que afecta a nervios periféricos y musculatura distal. Existen dos subtipos y frecuentemente los pacientes afectados por esta enfermedad requieren cirugías ortopédicas correctoras a lo largo de la vida. Existen reportes de bloqueos de nervio periférico en pacientes con CMT, en los cuales ninguno ha reportado exacerbación de los síntomas neurológicos. En éstos, se han reportado diferencias en la sensibilidad a los anestésicos locales y en el tiempo de duración del efecto. Ante la ausencia de mayor evidencia, no existe contraindicación formal en estos pacientes y se recomienda mantener precauciones en las dosis administradas y una técnica cuidadosa al momento de realizar el bloqueo ${ }^{(22)}$. 


\section{Neurofibromatosis}

La neurofibromatosis (NF) es una enfermedad autosómica dominante con dos subtipos (NF 1 y NF 2). La NF1 es la más frecuente y su forma histológica caracterizada por neurofibromas nodulares, puede afectar a los nervios periféricos con tumoraciones de diferentes tamaños. Existen reportes de bloqueos de nervio periférico en pacientes con NF1 y las principales preocupaciones están focalizadas en los pacientes que desarrollan neurofibromatomas nodulares en los nervios con indicación de bloqueo para un determinado procedimiento. Esto ha puesto como recomendación en estos pacientes incluir a las medidas mencionadas para otras patologías, el buscar activamente una localización anatómica a bloquear libre de tumores con ultrasonido $(19,22)$.

Miastenia Gravis

La Miastenia Gravis (MG) es un síndrome inmunológico que interfiere la normal despolarización de la motoneurona distal en la unión neuromuscular. En estos pacientes, el elevado riesgo de falla respiratoria postoperatoria, puede ser disminuido con el uso de anestesia regional. Hasta el momento, existen reportes de bloqueos de nervio periférico en pacientes con MG en distintos abordajes (axilar, infraclavicular y paravertebral) sin exacerbación de los síntomas neurológicos previos. Sin embargo, esto no excluye la necesidad de vigilar el desarrollo de una crisis miasténica en el perioperatorio aún si se han evitado los relajantes neuromusculares y la anestesia general $(19,22)$.

\section{Otras condiciones clínicas}

Las patologías neurológicas que afectan al nervio periférico son múltiples y muchas de ellas son infrecuentes al contexto perioperatorio. En la actualidad, existen escasos reportes de anestesia regional periférica en pacientes con Esclerosis lateral amioatrófica, daño previo por quimioterápicos, síndrome post poliomielitis y Guillain-Barré sin una clara exacerbación de los síntomas que contraindiquen su aplicación. En todas estas condiciones, se sugieren las precauciones antes mencionadas con el fin de minimizar un potencial riesgo de daño (19).

\section{Rechazo de la técnica}

El creciente uso de la anestesia regional en las últimas décadas ha despertado interés en los aspectos éticos y médicolegales de esta subespecialidad. El consentimiento informado puede ser entendido como un contrato bilateral entre el médico responsable de un procedimiento y el paciente o su tutor en determinados casos ${ }^{(29)}$. Esta entrega bilateral de información exige entre otras, la aceptación por parte del paciente y del médico de los beneficios y potenciales riesgos de las técnicas propuestas para cada caso. Las discusiones de consentimiento informado sobre estos procedimientos técnicos son necesarias para la protección de la autonomía del paciente y el creciente interés en este tópico a lo largo de los años, se ha centrado en reforzar la relación médico paciente protegiendo y sosteniendo este princi-

pio ético ${ }^{(30)}$. Está lejos de la intención de esta revisión profundizar en los alcances del consentimiento informado en anestesia regional. Sin embargo, es importante recalcar que la carta de derechos y deberes del paciente actualmente vigente en nuestro sistema de salud, permite al paciente aceptar o rechazar cualquier tratamiento en cualquier momento. Más importante aún, debido a las limitaciones de un entorno peri operatorio presionado por el tiempo, y el hecho de que el encuentro entre el anestesiólogo y el paciente ocurre en algunos casos sólo breves momentos antes del procedimiento planificado, nos plantea un enorme desafío para logar una discusión de consentimiento bien informada entre el especialista y el paciente, que permita a este último aceptar o declinar una técnica regional de manera adecuada.

\section{Anestesia Regional en pacientes bajo sedación profunda o Anestesia General}

Durante el desarrollo de la anestesia regional como importante técnica al cuidado de los pacientes en el periodo peri operatorio, es razonable plantear que se ha erguido como una técnica segura y efectiva realizada en anestesiólogos entrenados. En la práctica clínica, es cada vez más común recibir por parte de los pacientes exigencias relacionadas a la comodidad y confort durante el desarrollo de su cirugía, incluyendo la realización de un bloqueo de nervio periférico antes de someterse a una intervención quirúrgica; así no es extraño recibir solicitudes que implican "no sentir ni oír nada" ya sea por fobia o simple comodidad. Esto plantea la problemática de si es seguro realizar un bloqueo de nervio periférico bajo sedación profunda o anestesia general. Históricamente, podría considerarse casi una contraindicación formal el realizar un bloqueo en un paciente completamente anestesiado (31) sometiendo al paciente a 
una situación de disconfort que podría poner en jaque la experiencia final del paciente durante nuestra intervención, aumentando de manera importante su ansiedad peri operatoria.

Desde hace décadas, se pensaba que el contacto aguja-nervio producía inmediatamente dolor; bajo el clásico principio de la anestesia regional que "sin parestesia no hay anestesia", se ha mantenido en el ideario colectivo que la evocación de disestesia y por consiguiente dolor, constituyen un monitor inequívoco de una peligrosa aproximación entre la aguja y nervio o peor inclusive de una inyección intraneural no advertida, indistintamente de la técnica de neurolocalización utilizada. Sin embargo, numerosos estudios han puesto en duda este principio, puesto que se ha documentado daño neurológico transitorio o permanente en pacientes despiertos, quienes no evocaron dolor mientras se realizaba el procedimiento y solo un tercio de los pacientes evocó alguna respuesta tras el contacto directo de la aguja con el nervio ${ }^{(32)}$ lo que permite inferir a priori que el paciente es una monitorización con bajo rendimiento para complicaciones neurológicas durante un bloqueo de nervio periférico. Es razonable plantearse si la integración de nuevas tecnologías, como ultrasonidos de alta resolución nos permitan reconocer y delimitar mejor las estructuras (33) y así avanzar de manera más segura nuestra aguja respetado los límites anatómicos del nervio periférico.

A pesar de que la literatura no es suficientemente clara al respecto, es probable que la gran mayoría de los bloqueos de nervio periférico se realicen en pacientes plenamente despiertos o bajo sedación ligera. La ASRA mantiene sus recomendaciones elaboradas el año 2015 y $2018^{(7,26)}$ donde recomienda que los bloqueos de nervio periférico no se realicen de rutina bajo sedación profunda o anestesia regional, indicando incluso en versiones anteriores, la fuerte sugerencia de realizar un bloqueo interescalénico sólo en pacientes despiertos. Cabe señalar, que estas recomendaciones surgen a partir de un pequeño grupo de casos reportados de complicaciones neurológicas posteriores una anestesia con un plexo braquial interescalénico realizado sin ultrasonido. Por otro lado, las recomendaciones de seguridad e innovación técnica han evolucionado desde la elaboración de esas guías clínicas. Por último, es inevitable no mencionar al discutir este punto, las pocas aprehensiones que existen al momento de realizar un bloqueo de nervio periférico en la población pediátrica, donde inevitablemente se llevan a cabo bajo sedación profunda o anestesia general con la premisa del confort del paciente pediátrico y la escasa colaboración a la técnica que puede resultar una punción no sedado o anestesiado. En esta situación, estamos enfrentados prácticamente a los mismos riesgos teóricos y estructuras anatómicas potencialmente susceptibles que en la población adulta. Es razonable plantear entonces, que un bloqueo de nervio periférico realizado por un especialista entrenado y con las condiciones técnicas y tecnológicas adecuadas, pueda realizarse bajo anestesia general o sedación profunda anteponiendo siempre el beneficio sobre el potencial riesgo de daño neurológico en un paciente que así lo desee ${ }^{(34,35,36)}$.

\section{Anestesia Regional en entornos no seguros}

La anestesiología en los últimos años se ha posicionado como una de las especialidades líderes en lo que respecta a la seguridad del paciente, convirtiéndose en un modelo para otro tipo de disciplinas del área de la medicina. Desde el año 2000, se reconocen los esfuerzos y estrategias abordados por los anestesiólogos para disminuir los riesgos relacionados con factores humanos y sistémicos durante la práctica anestésica. Todo esto con el fin de lograr en las organizaciones sanitarias una práctica asistencial segura e institucionalizar a la vez la seguridad del paciente como tema de preocupación profesional ${ }^{(37)}$. En este sentido, la práctica de la anestesia regional periférica no ha quedado excluida. Con la implementación de las listas de verificación en el ámbito quirúrgico, impulsadas desde el 2009 por la Organización Mundial de la Salud, se consiguió en los últimos años una reducción de los incidentes de origen quirúrgicas, como la cirugía en el sitio equivocado. En 2011 el National Health Service (NHS) del Reino Unido, presentó una iniciativa nacional de seguridad del paciente llamado "Stop Before You Block", orientada a reducir la incidencia de bloqueo inadvertido del lado incorrecto durante la anestesia regional. Las alertas que impulsaron están campaña se sustentan principalmente en las eventuales consecuencias de los bloqueos periféricos, incluidas las complicaciones del bloqueo innecesario, como la lesión nerviosa y la toxicidad por anestésicos locales. En el peor de los casos este error puede llevar al equipo a continuar con la cirugía en el sitio equivocado ${ }^{(38)}$. Por la magnitud y el potencial de daño, la NHS incluyó en el 2015 a los bloqueos periféricos de sitio incorrecto como un "Never Events", es decir, incidentes que no deben ocurrir jamás dentro de la práctica asistencial ${ }^{(39)}$.

Si bien aún no existe claridad sobre el impacto real que estas iniciativas han tenido en la incidencia de bloqueos de sitios incorrectos, principalmente por la variabilidad en su aplicación ${ }^{(40)}$, si existe un consenso en que debe hac- 
erse una pausa y verificación antes de cualquier procedimiento anestésico regional periférico, verificando siempre las condiciones óptimas de seguridad para el paciente antes y durante el bloqueo de nervio periférico. Existe cierta certeza que la prevención de estos eventos debe darse en un contexto organizativo que garantice la seguridad por medio de protocolos y directrices que guíen la práctica del anestesiólogo apoyada por los líderes de cada institución

y realizada por todos los miembros del equipo ${ }^{(41)}$ y la falta de condiciones de seguridad al proceder con la anestesia regional periférica, puede plantear al anestesiólogo la posibilidad de diferir un bloqueo de nervio periférico en un entorno no seguro.

\section{Conclusiones}

La práctica de la anestesia regional periférica como un procedimiento seguro y con la menor incidencia de fallos, errores y consecuencias, está determinado por múltiples factores en donde convergen aspectos relacionados a características propias de los pacientes, del operador, del ambiente o entorno en donde se desarrolla, a las tecnologías e implementos técnicos disponibles y de la cultura sistémica de la organización o servicio que implementa esta práctica. La decisión de ejecutar esta técnica deberá ir acompañada de la evaluación objetiva por parte del profesional de cada uno de estos aspectos, con el objetivo de determinar si su realización ocurre en el contexto más seguro y óptimo para el paciente.

\section{Referencias}

1. Kopp, S.L. and Horlocker, T.T. Regional anaesthesia in day-stay and short-stay surgery. Anaesthesia. 2010; 65: 84-96. https://doi.org/10.1111/j.1365-2044.2009.06204.x

2. Brull, R., McCartney, C. J. L., Chan, V. W. S., \& El-Beheiry, H. (2007). Neurological complications after regional anesthesia: Contemporary estimates of risk. Anesthesia and Analgesia. 2007; 104(4), 965-974. https:// doi.org/10.1213/01.ane.0000258740.17193.ec

3. Kaushal M, Rubin RE, Kaye AD, Gritsenko K. Anticoagulation and Neuraxial/Peripheral Anesthesia. Anesthesiol Clin. 2017;35(2):e21-39. http://dx.doi.org/10.1016/j.anclin.2017.01.002

4. Vandermeulen E. Regional anaesthesia and anticoagulation. Best Pract Res Clin Anaesthesiol. 2010;24(1):121-31. http://dx.doi.org/10.1016/j.bpa.2009.09.004

5. Carles M, Beloeil H, Bloc S, Nouette-Gaulain K, Aveline C, Cabaton J, et al. Anesthésie locorégionale périnerveuse. Anesthésie \& Réanimation. 2017;3(2):198-206. http://dx.doi.org/10.1016/j.anrea.2017.01.002

6. Gogarten W, Vandermeulen E, Van Aken H, Kozek S, Llau J V., Samama CM. Regional anaesthesia and antithrombotic agents: Recommendations of the European Society of Anaesthesiology. Eur J Anaesthesiol. 2010;27(12):9991015. http://dx.doi.org/10.1097/EJA.0b013e32833f6f6f.

7. Horlocker TT, Vandermeuelen E, Kopp SL, Gogarten W, Leffert LR, Benzon HT. Regional Anesthesia in the Patient Receiving Antithrombotic or Thrombolytic Therapy: American Society of Regional Anesthesia and Pain Medicine Evidence-Based Guidelines (Fourth Edition). Vol. 43, Regional Anesthesia and Pain Medicine. 2018. 263309 p. http://dx.doi.org/10.1097/AAP.0000000000000763.

8. Godier A, Fontana P, Motte S, Steib A, Bonhomme F, Schlumberger S, et al. Management of antiplatelet therapy in patients undergoing elective invasive procedures. Proposals from the French Working Group on perioperative haemostasis (GIHP) and the French Study Group on thrombosis and haemostasis (GFHT). Anaesth Crit Care Pain Med. 2018;37(4):379-89. http://dx.doi.org/ 10.1016/j.accpm.2017.12.012

9. Tsui BCH, Kirkham K, Kwofie MK, Tran DQ, Wong P, Chin KJ, et al. Practice advisory on the bleeding risks for peripheral nerve and interfascial plane blockade: evidence review and expert consensus. Can J Anesth 2019;66(11):1356-84. https://doi.org/10.1007/s12630-019-01466-w

10. Vela Vásquez RS, Peláez Romero R. Aspirin and spinal haematoma after neuraxial anaesthesia: Myth or reality? Br J Anaesth. 2015;115(5):688-98. http://dx.doi.org/10.1093/bja/aev348

11. Volk T, Kubulus C. New oral anticoagulants and neuraxial regional Anesthesia. Curr Opin Anaesthesiol. 2015;28(5):605-9. http://dx.doi.org/10.1097/AC0.0000000000000234

12. Benzon HT, Avram MJ, Green D, Bonow RO. New oral anticoagulants and regional anaesthesia. Br J Anaesth 2013;111(SUPPL.1):i96-113. http://dx.doi.org/10.1093/bja/aet401 
13. Hewson DW, Bedforth NM, Hardman JG. Peripheral nerve injury arising in anaesthesia practice. Anaesthesia. 2018;73:51-60. http://dx.doi.org/10.1111/anae.14140

14. Franco CD, Sala-Blanch X. Functional anatomy of the nerve and optimal placement of the needle for successful (and) safe nerve blocks. Curr Opin Anaesthesiol. 2019;32(5):638-42. http://dx.doi.org/10.1097/ ACO.0000000000000776

15. Liguori GA. Complications of Regional Anesthesia: Nerve Injury and Peripheral Neural Blockade. J Neurosurg Anesthesiol. 2004;16(1):84-6. http://dx.doi.org/10.1097/00008506-200401000-00018

16. Sondekoppam R V., Tsui BCH. Factors associated with risk of neurologic complications after peripheral nerve blocks: A systematic review. Anesth Analg. 2017;124(2):645-60. http://dx.doi.org/10.1213/ANE.00000000000001804

17. Lam KK, Soneji N, Katzberg H, Xu L, Chin KJ, Prasad A, et al. Incidence and etiology of postoperative neurological symptoms after peripheral nerve block: a retrospective cohort study. Reg Anesth Pain Med. 2020;45(7):495504. http://dx.doi.org/10.1136/rapm-2020-101407

18. Chui J, Murkin JM, Posner KL, Domino KB. Perioperative peripheral nerve injury after general anesthesia: A qualitative systematic review. Anesth Analg. 2018;127(1):134-43. http://dx.doi.org/10.1213/ANE.0000000000003420

19. Kopp SL, Jacob AK, Hebl JR. Regional Anesthesia in Patients with Preexisting Neurologic Disease. Reg Anesth Pain Med. 2015;40(5):467-78. http://dx.doi.org/ 10.1097/AAP.0000000000000179

20. Watson JC, Huntoon MA. Neurologic Evaluation and Management of Perioperative Nerve Injury. Reg Anesth Pain Med. 2015;40(5):491-501. http://dx.doi.org/10.1097/AAP.0000000000000185

21. Ten Hoope W, Looije M, Lirk P. Regional anesthesia in diabetic peripheral neuropathy. Curr Opin Anaesthesiol. 2017;30(5):627-31. http://dx.doi.org/10.1097/ACO.0000000000000506

22. McSwain JR, Doty JW, Wilson SH. Regional anesthesia in patients with pre-existing neurologic disease. Curr Opin Anaesthesiol. 2014;27(5):538-43. http://dx.doi.org/10.1097/AC0.0000000000000107

23. Rigaud M, Filip P, Lirk P, Fuchs A, Gemes G, Hogan Q. Guidance of block needle insertion by electrical nerve stimulation: a pilot study of the resulting distribution of injected solution in dogs. Anesthesiology. 2008 Sep;109(3):473-8. http://dx.doi.org/10.1097/ALN.0b013e318182af0b.

24. Bigeleisen PE, Moayeri N, Groen GJ. Extraneural versus intraneural stimulation thresholds during ultrasound-guided supraclavicular block. Anesthesiology. 2009 Jun;110(6):1235-43. http://dx.doi.org/10.1097/ ALN.0b013e3181a59891

25. Keyl C, Held T, Albiez G, Schmack A, Wiesenack C. Increased electrical nerve stimulation threshold of the sciatic nerve in patients with diabetic foot gangrene: a prospective parallel cohort study. Eur J Anaesthesiol. 2013 Jul;30(7):435-40. http://dx.doi.org/10.1097/EJA.0b013e328360bd85

26. Neal JM, Barrington MJ, Brull R, Hadzic A, Hebl JR, Horlocker TT, et al. The Second ASRA Practice Advisory on Neurologic Complications Associated with Regional Anesthesia and Pain Medicine: Executive Summary 2015. Reg Anesth Pain Med. 2015;40(5):401-30. http://dx.doi.org/10.1097/AAP.0000000000000286

27. Koff MD, Cohen JA, McIntyre JJ, Carr CF, Sites BD. Severe brachial plexopathy after an ultrasound-guided single-injection nerve block for total shoulder arthroplasty in a patient with multiple sclerosis. Anesthesiology. 2008 Feb;108(2):325-8. http://dx.doi.org/10.1097/01.anes.0000299833.73804.cd

28. Finucane BT, Terblanche OC. Prolonged duration of anesthesia in a patient with multiple sclerosis following paravertebral block. Can J Anaesth. 2005 May;52(5):493-7. http://dx.doi.org/10.1007/BF03016529.

29. Bertini L, Savoia G, De Nicola A, Ivani G, Gravino E, Albani A, Alemanno F, Barbati A, Borghi B, Borrometi F, Casati A, Celleno D, Ciaschi A, Corcione A, De Negri P, Di Benedetto P, Evangelista M, Fanelli G, Grossi P, Loreto M, Margaria E, Mastronardi P, Mattia C, Nicosia F, Nolli M, Rutili A, Santangelo E, Sucre J, Tagariello V, Varrassi G, Paoletti F, Tufano R; SIAARTI. SIAARTI guidelines for safety in locoregional anaesthesia. Minerva Anestesiol. 2006 Sep;72(9):689-722.

30. Tierney S, Perlas A. Informed consent for regional anesthesia. Curr Opin Anaesthesiol. 2018;31(5):614-21. http:// dx.doi.org/0.1097/ACO.0000000000000635 
31. Benumof J; Permanent Loss of Cervical Spinal Cord Function Associated with Interscalene Block Performed under General Anesthesia. Anesthesiology 2000;93(6):1541-1544.

32. Perlas A, Niazi A, McCartney C, Chan V, Xu D, Abbas S. The sensitivity of motor response to nerve stimulation and paresthesia for nerve localization as evaluated by ultrasound. Reg Anesth Pain Med. 2006 Sep-Oct;31(5):445-50. http://dx.doi.org/10.1016/j.rapm.2006.05.017

33. Varela V, Ruíz C, Pomés J, Pomés I, Montecinos S, Sala-Blanch X. Usefulness of high-resolution ultrasound for small nerve blocks: visualization of intercostobrachial and medial brachial cutaneous nerves in the axillary area. Reg Anesth Pain Med. 2019 Aug 26:rapm-2019-100689. http://dx.doi.org/10.1136/rapm-2019-100689.

34. Marhofer P. Regional blocks carried out during general anesthesia or deep sedation: Myths and facts. Curr Opin Anaesthesiol. 2017;30(5):621-6. http://dx.doi.org/10.1097/ACO.0000000000000504

35. Helander EM, Kaye AJ, Eng MR, Emelife PI, Motejunas MW, Bonneval LA, et al. Regional Nerve Blocks -Best Practice Strategies for Reduction in Complications and Comprehensive Review. Curr Pain Headache Rep. 2019;23(6). http://dx.doi.org/10.1007/s11916-019-0782-0

36. Barrington MJ, Uda Y. Did ultrasound fulfill the promise of safety in regional anesthesia? Curr Opin Anaesthesiol. 2018;31(5):649-55. http://dx.doi.org/10.1097/ACO.0000000000000638

37. Gaba DM. Anaesthesiology as a model for patient safety in health care. BMJ. 2000 Mar 18;320(7237):785-8. http://dx.doi.org/10.1136/bmj.320.7237.785

38. Mellin-Olsen J, Staender S, Whitaker DK, Smith AF. The Helsinki Declaration on Patient Safety in Anaesthesiology. Eur J Anaesthesiol. 2010 Jul;27(7):592-7 https://doi.org/10.1097/EJA.0b013e32833b1adf

39. Barrington MJ, Uda Y, Pattullo SJ, Sites BD. Wrong-site regional anesthesia: review and recommendations for prevention? Curr Opin Anaesthesiol. 2015 Dec;28(6):670-84 http://dx.doi.org/10.1097/ACO.0000000000000258

40. Neal JM. Ultrasound-guided regional anesthesia and patient safety: Update of an evidence-based analysis. Reg Anesth Pain Med. 2016;41(2):195-204. http://dx.doi.org/10.1097/AAP.0000000000000295

41. Deutsch ES, Yonash RA, Martin DE, Atkins JH, Arnold T V., Hunt CM. Wrong-site nerve blocks: A systematic literature review to guide principles for prevention. J Clin Anesth. 2018;46:101-11. https://dx.doi.org/10.1016/ j.jclinane.2017.12.008 
CARSACH

Página 65 